\title{
Pediatric interventional radiology: a maturing subspecialty
}

\author{
James S. Donaldson ${ }^{1}$
}

Received: 19 December 2016/Accepted: 2 February 2017 / Published online: 6 March 2017

(C) Springer-Verlag Berlin Heidelberg 2017

The 2015 survey published in this issue titled "Pediatric Interventional Radiology Workforce Survey: 10-year follow-up" reveals what many of us hoped would be growth and a maturing of pediatric interventional radiology (PIR) [1].

Charles Dotter, recognized as the "father of interventional radiology," performed the first angioplasty of a stenotic iliac artery in 1964 [2, 3]. This historic event introduced changes over a 20 - to 30 -year timeframe that saw interventional radiology (IR) become a legitimate and essential subspecialty in adult health care. For many reasons, however, children were mostly left out during this subspecialty evolution.

As the early pioneers in IR invented catheters, wires, snares, baskets and coils, most devices were too large to even consider using in a child. And even if they could be used in a child, which pediatrician would recommend and which parent would consent to an experimental procedure on their child? But as IR matured and outcomes proved its effectiveness in adults, one would have thought innovations would quickly bring new techniques to children. But it wasn't that easy and it didn't much happen. Interventional radiologists weren't trained on children, catheters were still too large and unwieldy, children wouldn't hold still, and back then anesthesiologists disliked radiology departments. Additionally, the demand was not there from clinical staff — in some circumstances, one might say there was aggressive resistance, e.g., "children are

James S. Donaldson

jdonaldson@luriechildrens.org

1 Department of Medical Imaging,

Ann \& Robert H. Lurie Children's Hospital of Chicago,

Feinberg School of Medicine at Northwestern University,

225 E. Chicago Ave.,

Chicago, IL 60611-2991, USA not just small adults and just because it works in adults...." It was the rare pediatric radiologist who was willing to confront a pediatric surgeon and say "there is another way to do this." Pediatric interventional radiology (PIR) was left behind.

In the early 1990s, through networking of some frustrated members of the Society of Interventional Radiology (SIR) and the Society for Pediatric Radiology (SPR), an interest in pediatric interventional radiology began to grow. But it's not easy to grow from nothing, with no formal training pathway and no one to show the way. Some pediatric radiologists were successful in pulling themselves up by their bootstraps and convincing colleagues that there really was a better way. Some adult interventional radiologists saw the large gap and began making the extra effort to provide previously unavailable procedures to children. Children's hospitals were asked to invest in equipment and provide staffing in good faith believing that "if you build it, they will come." The hardest part: changing the established practice habits of referring physicians. This required persuading them to trust their patients to new procedures performed by new and often very young radiologists. Those were the early years of PIR.

Finally, 20+ years after IR swept the adult world, PIR at many pediatric institutions has developed into a subspecialty where pediatricians, pediatric surgeons and subspecialists rely on interventional radiology as an essential part of patient care - 24/7/365. More than once I have been asked by the graduating pediatric surgery fellow, "what will I do without PIR at my new job?" Now there is a demand.

The current workforce survey compares the subspecialty growth from 2005 to now and reveals that the culture has changed, there is demand from clinicians, and PIR has grown $[1,4]$. The number of pediatric institutions where PIR is available has grown by $131 \%$. The number of practicing pediatric interventional radiologists with adult IR or dedicated pediatric IR fellowships has increased. The numbers, types and 
complexity of cases performed have all grown. Pediatric IR has also extended well beyond North America. The Society of Pediatric Interventional Radiology (SPIR), supported by both the SPR and SIR, was organized in 2009 and now has nearly 250 members from 55 countries. It is encouraging to see new radiology graduates become interested in PIR and continue the evolution and maturation of this exciting subspecialty.

There remain many gaps in PIR coverage. Some hospitals in North America and even entire countries have limited or no access to PIR. With the passion of many SPIR members and continued support from the SPR and SIR this subspecialty will follow the growth and maturation seen in adult IR. I look forward to seeing the next 10-year survey.

A special thanks to Dr. C. Kaufman and co-authors for taking time to conduct this survey and show us the exciting progress.
Compliance with ethical standards

Conflicts of interest None

\section{References}

1. Kaufman C, James C, Harned R et al (2017) Pediatric interventional radiology workforce survey: 10-year follow-up. Pediatr Radiol. doi: 10.1007/s00247-017-3796-y

2. Dotter CT, Judkins MP (1964) Transluminal treatment of arteriosclerotic obstruction: description of a new technique and preliminary report of its application. Circulation 30:654-670

3. Keller FS, Rosch J (1994) A personal memoir of Charles Dotter. In: The father of interventional radiology, Charles Dotter: highlights of his life and research. Excerpta Medica Publishers, Tokyo, pp. 7-9

4. Sidhu MK, James CJ, Harned RK et al (2007) Pediatric interventional radiology workforce survey summary. Pediatr Radiol 37:113-115 